\title{
Designing and Constructing Smart T-shirt for Prevention from Incidence and Recurrence of Lumbar Herniated Disc
}

Behnam Amini Daghalian ${ }^{1}$, Hassan Amini ${ }^{1}$, Seyed Mahdi Vahid Pakdel ${ }^{2}$, Farahnaz Varmazyar ${ }^{1}$, Farid Gharibi $^{3^{*}}$, Amin Vahdati ${ }^{4}$, Reza Dehghan ${ }^{5}$

1. Students' Research Committee, Tabriz University of Medical Science, Tabriz, Iran

2. Department of Prosthodontics, Tabriz University of Medical Sciences, Tabriz, Iran

3. Students' Research Committee, Tabriz University of Medical Science, Tabriz, Iran

4. Tabriz University of Medical Sciences

5. Shahrdari University of Applied Science, Tabriz, Iran

\begin{abstract}
Inappropriate positions of body in daily activities can lead to several problems in people health and impose great direct and indirect costs on different sectors of society. The purpose of this research was to design and construct a smart T-shirt for prevention from incidence and recurrent of lumbar herniated disc (LHD). The plan of this T- shirt was provided using a number of tensile or stretch arms on T- shirt for identifying the inappropriate moves of the body and then associating them to a vibration system for alarming the individual. This cover has the ability to prevent from many problems of backbone, especially LHD. This innovation has several advantages including decline in many problems regarding backbone or spinal cord and chronic pain in such areas, decreasing in treatment costs, growing in productivity capacity of people and society, and high chance of product to mass production. Thanks to the cost-effectiveness of this T-shirt, its use is strongly recommended.
\end{abstract}

Keywords: Smart T-shirt; Prevention; Lumbar Herniated Disc

\section{Introduction}

Diseases related to backbone and their personal and social adverse effects are always considered as one of the main health concerns such that, even passing of the time and technology developments cannot eliminate its destructive effects. One of the main diseases in this area, which affects different levels of the society and leaves many negative effects on physical, economic and social conditions, is LHD
(Lumbar Herniated Disc). In many cases, this disease can lead to complicated surgical operations (1). The greatest prevalence of LHD is seen among people with 40-45 years old and usually affects intervertebral disc L4-L5 and L5$\mathrm{SI}$, as $90 \%$ of cases are related to these areas (2). Evidences show that $80-90 \%$ of LHD operations can result in good outcomes in first year after doing such surgeries, due to enhance

\section{Corresponding author:}

Farid Gharibi

Students' Research Committee, Tabriz University of Medical Science, Tabriz, Iran

Mobile: +989181332935 E-mail: gharibihsa@gmail.com

Receive date: 2015-06-29| Accept date: 2015-07-18| Publish date: 2015-08-04

DOI: 10.7575/aiac.abcmed.15.03.04.07

\section{A. I}


and development of treatment and surgical methods (3).

Surgical methods for LHD have significant effectiveness and can lead to suitable improvement. Nevertheless, in $10-15 \%$ of cases some inappropriate results can be seen and sciatica and back pain are sometimes continued $(4,5)$. Inappropriate recognitions before operation, unsuitable election of surgical candidate, inappropriate planning to operation and incomplete surgical operations can lead to defects (6).

The main point is that, after operation and treatment, remarkable part of these cases reoccurs and patients face several problems. Recurrent herniated lumber disc is one of common causes of disability and failed operations, which are seen in $5-15 \%$ of the cases (7-9). The recurrent events have been reported in $3.8-7.4 \%$ in the previous disc angle and position $(9,10)$.

The definitions of recurrent LDH proposed by researchers are very different. However, most of them has defined the disease as "an LHD which is created in previous loci and vertebrae after at least 6 months without any pain" $(11,12)$. This event is a pathological process which can lead to disability (13). According to the reports of researchers, $25 \%$ of patients experience next operations after a short period from the first operation (14).

In a study, $28 \%$ of patients experienced pain in feet and back, 12 years after operation (15) which ultimately led to pain, disability and reoperation (16). The findings of previous studies show that the symptoms, severity and disability level in recurrent LDH and primary $\mathrm{LDH}$ are very similar (17) and the total rate of repeated operations in these patients have been reported to be $5.2-12 \%(9,10)$.

In addition, low back pain, which is the main cause of disability in the United States and the second cause of referring to the clinicians among people below 45, has been considered as one of the destructive effects of LDH (2). The annual rate of occurrence and prevalence rate of back pain in all life period are $5 \%$ (18) and $80 \%$, respectively (19). Yorimitsu and colleagues showed that young people below 35 , who are in best period of activity and production, suffer from severe pains after operations (20). This trend can show further social and economic destructive effects of this event.

The most valid statistics have been recently released by Spine Patient Outcome Research Trial (SPORT). These information indicate that second operations take place within the first year after operation ( $6 \%$ of patients), second year after operation ( $8 \%$ of patients), third year after operation (9\%) and forth year after operation (4\%), half of which are due to recurrent $\operatorname{LDH}(21,22)$.

The evidences have shown that the risk factors in recurrent LHD and even primary LHD are: defect or disability of disc itself, continual exposure to stresses from lifting things, shake or vibration, overweight and obesity, ageing, smoking, diabetes, size and height of disc, and the visibility level of hernia while operation (16, 23).

Hard and heavy activities are considered as a predictive variable in the reoccurrence of this disease, because these works require lifting objects repeatedly and also, repeated shake can have significant effect on this recurrence $(16,23)$. Kara et al. described inappropriate and unusual activity and nonscientific movements as important and effective factors in recurrence and repeated operations (24). Positions rang of the person in situations like sitting, walking, bending or sleeping can lead to this event or its resonating through unusual tensions or rotations. Thus, the main purpose of this study was to design and construct a smart T-shirt in order to prevent LHD and its recurrence. The Tshirt can identify inappropriate tensional 
movements and, then, aware the person. The researchers declared that commercialization and extensive use of this innovation will be an effective step in preventing people from inappropriate movements, and thereby it would prevent from occurrence and reoccurrence of $\mathrm{LDH}$, and also enhance health and quality of life.

\section{Materials and methods}

Design of the T-shirt

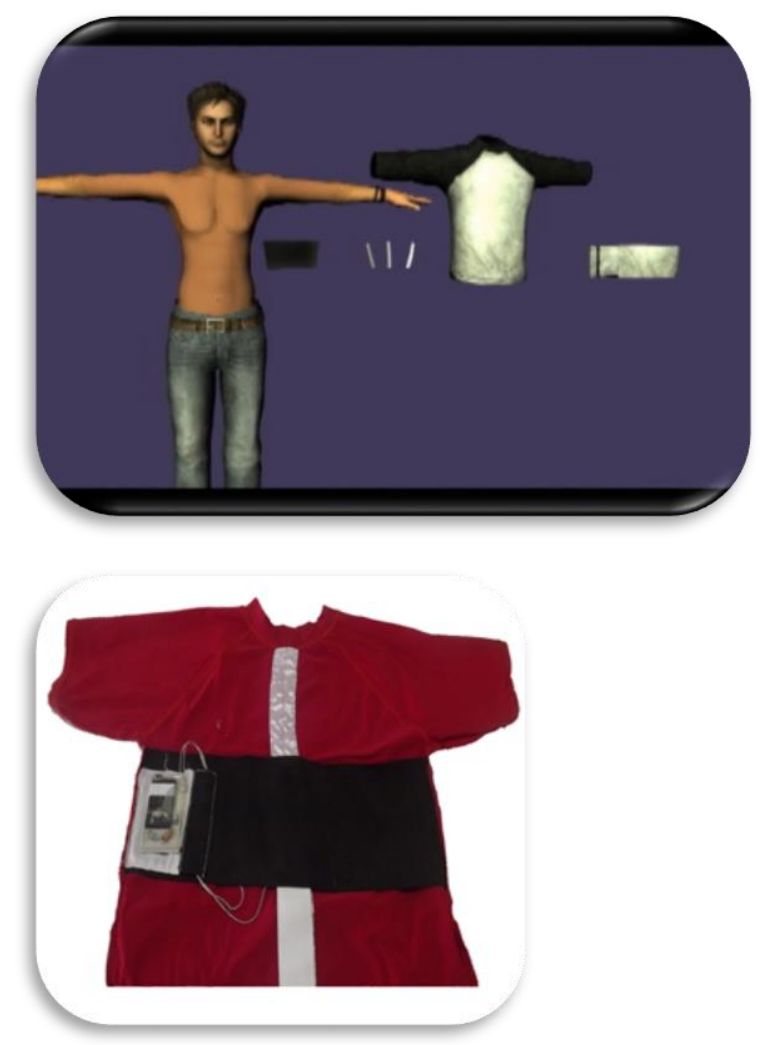

Figure 1

The T-shirt is based on 3 essential techniques. There is a wiring arm on middle of the T-shirt in back; it will brace and activate while improper sitting or false lifting and automatically vibrate to alert the user. Next part is a back brace which will hold rods protecting back from straining inappropriate pressures while hiking or prolonged sitting. Final part consists of wide abdominal elastic band in order to keep fitness and help slimming (Figure 1).

The structure and components of this T-shirt are as below: The system has been designed in the shape of a belt with four stretch or tensional arms. These arms are located in the back area such that two arms from sides are tied through abdomen. The cross shape of arms can be seen in the back side of the person. There is an electrical conductor wire inside these arms that reach together in a several points and these connected points have been related to a vibration system to alarm and aware the person (Figure 2).

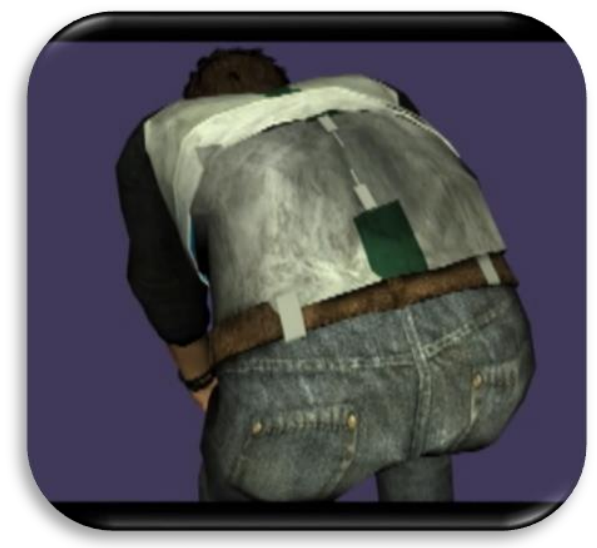

Figure 2

\section{Performance and technical capacities of the T-shirt}

The person can be warned through stress of the stretch or tensional arms which will activate vibration system for all inappropriate anatomical positions including sitting, bending, sleeping or any physical activity. This system, due to its rotation around the back and its protection of skeletal and muscular system of the back, is beneficial, even when lifting objects and doing other activities, and can prevent from excessive pressures to back and probable damages to its tissues (Figure 3 ). 


\section{Results and discussion}

Researchers argue that proper use of this Tshirt, that has unique characteristics, can have many benefits for patients, health system and society. Regarding the fact that main part of LDH cases, especially in back area, is related to the inappropriate anatomical positions in daily

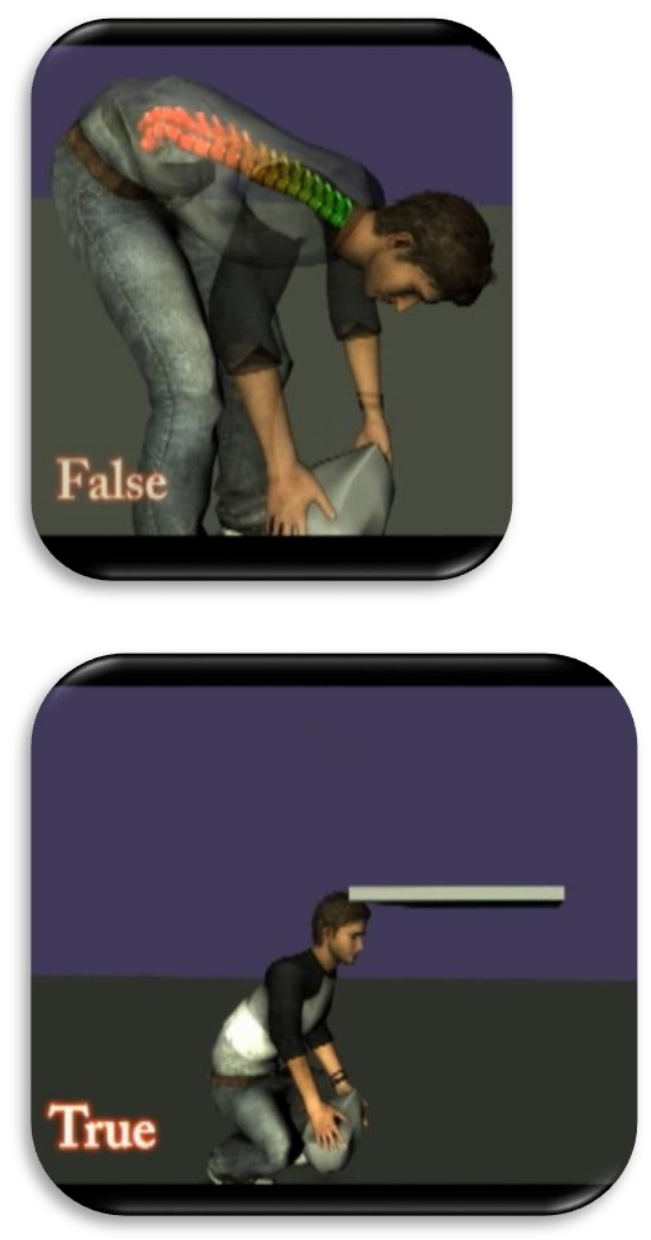

\section{Figure 3}

life or wrong and heavy activities in work place. Thus, this system, through effective and timely recognizing inappropriate positions and then giving alarm, can be successful in preventing incorrect bending to pick up objects, and thereby in correcting these positions. Therefore, the rate of this disease both in primary and secondary (cases of recurrence) forms can be acceptably reduced, which in turns can lead to decreased burden of the disease and treatment costs to person, families, health system and society.

On the other hand, due to the fact that these diseases are often seen in people at their age of 25-45 and have many adverse effects on physical capacities and abilities and even can cause to permanent defects and loss of productivity, using this T-shirt can be beneficial in preventing from such problems and damages to family and society. Decrease in the rate of absence from the work originating from these temporally or permanent defects and increase in productivity and economical potential are the other benefits that would result from this technology.

Moreover, this innovation, through correcting the physical positions of the individuals, can have significant effect on preventing chronic pain of the back, especially low back pain. It should be said that this T-shirt, with its special mechanism of action, can prevent effectively from other problems that are the result of inappropriate anatomical positions and movements of the person, such as, lordosis, kyphosis and scoliosis.

Undoubtedly, one of the important criteria for suitability of an invention is its reasonable cost of product, which is considered in planning of this T-shirt. This T-shirt has been produced with low cost, and the technology used in it allows us to decrease the total price of its production. Its technology is not a complicated one; yet, it has a simple, effective and efficient mechanism of action. This technology can be produced in large scales.

Advantages:

O Avoiding chronic lower back pains in long term sitting

o Avoiding the risk of lumber disc

o Slimming and fitness

o Preventing from high pressure to the spine 
o Modifying risky behaviors of picking up objects from the floor

o Preventing from hunched bac.

o The low cost of manufacturing and marketing

o Increasing efficiency at workplace

o Preventing premature fatigue at workplace

\section{Conclusion}

Smart T-shirt can prevent from problems related to backbone, and especially LDH with its recurrence. It also has significant physical, social and economic effects on community parts. Therefore, authors strongly recommend it for those with backbone problems, also individuals who have heavy and too physical activities. Furthermore, everyone can take advantage of this invention to avoid experiencing such diseases and their side effects.

\section{Acknowledgements}

The authors would like to express their gratitude to the Deputy of Researches and Technology of Tabriz University of Medical Sciences for their intellectual and financial supports.

\section{References}

1. Swartz KR, Trost GR. Recurrent lumbar disc herniation. Neurosurg Focus. 2003;15 (3):1-4.

2. Shahbandar L, Press J. Diagnosis and Nonoperative Management of Lumbar Disk Herniation Oper Tech Sports Med. 2005;13:114-21.

3. Hanley EN, Shapiro DE. The development of low-back pain after excision of a lumbar disc. J Bone Joint Surg 1989;71:719-21.

4. Dai LY, Zhou Q, Yao WF, Shen L. Recurrent lumbar disc herniation after discectomy: outcome of repeat discectomy. Surgical Neurology. 2005;64:226-31.

5. Hu RW, Jaglal S, Axcell T, Anderson G. A population-based study of reoperations after back surgery. Spine 1997;22:2265- 71.

6. Keskimaki I, Seitsalo S, Osterman H, Rissanen P. Reoperations after lumbar disc surgery: a population-based study of regional and interspecialty variations. Spine 2000;25:1500- 8.

7. Gaston P, Marshall RW. Survival analysis is a better estimate of recurrent disc herniation. J Bone Joint Surg Br 2003;85:535-7.

8. Mobbs RJ, Newcombe RL, Chandran KN. Lumbar discectomy and the diabetic patient: Incidence and outcome. J Clin Neurosci. 2001;8:10-3.

9. Suk KS, Lee HM, Moon SH, Kim NH. Recurrent lumbar disc herniation: Results of operative management. Spine 2001;26:672-76.

10. Atlas SJ, Leller RB, Chang Y, Deyo RA, Singer DE. Surgical and nonsurgical management of sciatica secondary to a lumbar disc herniation: Five-year outcomes from the marine lumbar spine study. Spine. 2001;26:1179-87.

11. Cinotti G, Gumina S, Giannicola G, Postacchini F. Contralateral recurrent lumbar disc herniation: Results of discectomy compared with those in primary herniation. Spine 1999;24:800-6.

12. Erbayraktar S, Acar F, Tekinsoy B. Outcome analysis of reoperations after lumbar discectomies: a report of 22 patients. Kobe J Med Sci. 2002;48:33-41. 
13. Glenn JS, Yaker J, Guyer RD, Ohnmeiss D. Anterior discectomy and total disc replacement for three patients with multiple recurrent lumbar disc herniations. The Spine Journal. 2011;11 1-6.

14. Atlas SJ, Keller RB, Wu YA. Long-term outcomes of surgical and nonsurgical management of sciatica secondary to a lumbar disc herniation: 10 year results from the Maine lumbar spine study. Spine. 2005;30:927-35.

15. Loupasis GA, Stamos K, Katonis PG. Seven- to 20-year outcome of lumbar discectomy. Spine 1999;24:2313-7.

16. Shimia M, Babaei-Ghazani A, Sadat BE, Habibi B, Habibzadeh A. Risk factors of recurrent lumbar disk herniation. Asian J Neurosurg 2013;8:93-6.

17. Jonsson B, Stroqvist B. Clinical characteristics of recurrent sciatica after lumbar discectomy. Spine 1996;21:500-5.

18. Herring SA, Weinstein SM. Assessment and nonsurgical management of athletic low back injury, in Nicholas JA, Hershman EB (eds): The Lower Extremity and Spine in Sports Medicine. Mosby; 1995.

19. Kaul M, Herring SA. Rehabilitation of lumbar spine injuries, in Kibler WB, Herring SA, Press JM, et al (eds): Functional Rehabilitation of Sports and Musculoskeletal Injuries. Gaithersburg: Aspen Publishers; 1998.

20. Yorimitsu E, Chiba K, Toyama Y, Hirabayashi K. Long-term outcomes of standard discectomy for lumbar disc herniation: a followup study of more than 10 years. Spine 2001;26:652-7.

21. Weinstein JN, Lurie JD, Tosteson TD, Tosteson AN, Blood E, Abdu WA, et al. Surgical versus non-operative treatment for lumbar disc herniation: four-year results for the Spine Patient Outcome Research Trial (SPORT). Spine. 2008;33:2789-800.

22. Aizawa T, Ozawa H, Kusakabe T, Nakamura T, Sekiguchi A, Takahashi A, et al. Reoperation for recurrent lumbar disc herniation: a study over a 20-year period in a Japanese population. J Orthop Sci 2012;17:107-13.

23. Mundt DJ, Kelsey JL, Golden AL, Pastides H, Berg AT, Sklar J. An epidemiologic study of non-occupational lifting as a risk factor for herniated lumbar intervertebral disc. Spine 1993;18:595-602.

24. Kara B, Tulum Z, Acar U. Functional results and the risk factors of reoperations after lumbar disc surgery. Eur Spine J 2005;14:43-8. 